\title{
Decision-Making in Transcatheter Edge-to-Edge Repair: Insights into Atrial Functional Mitral Regurgitation
}

\author{
Joon Bum Kim, M.D., Ph.D. \\ Department of Thoracic and Cardiovascular Surgery, Asan Medical Center, University of Ulsan College of Medicine, Seoul, Korea
}

\section{ARTICLE INFO}

Received June 17, 2021

Accepted August 5, 2021

Corresponding author

Joon Bum Kim

Tel $82-2-3010-5416$

Fax 82-2-3010-6966

E-mail jbkim1975@amc.seoul.kr ORCID

https://orcid.org/0000-0001-5801-2395

\begin{abstract}
The 2020 American College of Cardiology focused update on the mitral regurgitation (MR) pathway provides an excellent summary of the decision-making trees in the treatment of severe MR, in which 2 main branches of the flowchart are suggested depending on whether MR is primary or secondary. Surgery is suggested as preferable over transcatheter edge-to-edge repair (TEER) in primary MR that needs intervention. The decision-making for secondary MR generally prioritizes TEER over surgery according to the guidelines, but further stratification is necessary based on the pathophysiologic mechanisms of MR. TEER is probably the more suitable option in secondary MR caused by left ventricular dysfunction or dilatation, given the high perceived surgical risks, despite the lack of sufficient evidence in support of overt clinical benefits from surgical therapy in these patients. In atrial functional MR associated with atrial fibrillation (AF), however, concomitant ablation of AF seems to be a desirable option, as it has been demonstrated to be a key factor leading to improved survival, reduced stroke risk, and more durable mitral and tricuspid function in patients undergoing mitral surgery. Therefore, atrial functional MR requiring intervention may be best treated by surgical therapy that combines mitral repair and AF ablation in the majority of patients. This particular issue, however, needs further research to obtain scientific evidence to guide optimal management strategies.
\end{abstract}

Keywords: Transcatheter edge-to-edge repair, MitraClip, Mitral repair, Surgery, Atrial functional mitral regurgitation

\section{Introduction}

Two recent multi-center randomized trials have demonstrated that transcatheter edge-to-edge repair (TEER) is an effective treatment for primary mitral regurgitation (MR) as compared with surgical therapy and that it also improves survival in well-selected patients with functional MR with reduced left ventricular (LV) function in comparison to guideline-directed medical therapy $[1,2]$. These landmark clinical trials opened the era of transcatheter mitral valve $(\mathrm{MV})$ therapies for the treatment of severe symptomatic MR at the time of the consecutive triumphs of transcatheter aortic valve replacement (TAVR) for severe aortic stenosis, as TAVR was first performed for high-risk/ inoperable patients, and its indications then expanded to include low-risk patients, with overwhelmingly excellent outcomes [3-6]. As with TAVR, the demonstrated effective- ness of TEER may be just a glimpse of the widespread expansion of its applications in the future. This possibility is welcome from the perspectives of both the surgical and medical communities, as TEER would be a valuable addition to the options available to treat patients suffering from severe MR presenting with diverse baseline characteristics. However, TEER is not merely a possibility for the future; instead, it is already becoming a part of our daily practice, as we have seen a surging trend in its use in recent years [7].

The promising clinical results of this emerging transcatheter therapy in heart valve diseases have led to urgent calls to update practice guidelines so that they reflect the most up-to-date scientific evidence, and TEER is one of the core issues in these discussions [8,9]. The 2020 American College of Cardiology focused update on the MR pathway provides an excellent summary of the decision-making trees in the treatment of severe MR, in which 2 main branch- 
es of the flowchart are suggested depending on whether MR is primary or secondary [8].

\section{TEER versus surgery in primary mitral regurgitation}

For primary MR, the updated consensus considers surgical therapy to be the more preferable option, saving TEER only for patients with prohibitive surgical risk. This recommendation is principally grounded in the results of a randomized controlled trial (the Endovascular Valve Edgeto-Edge Repair Study [EVEREST] II trial) [1]. Despite the proven superiority of TEER over MV surgery in terms of lower rates of blood-product transfusion and prolonged ventilation, TEER was associated with a higher reintervention rate for MV dysfunction (20\% versus $2 \%, \mathrm{p}<0.001)$ [1]. With regard to other major safety measures, no significant differences were found between the TEER and surgery groups in the rates of myocardial infarction, the requirement for urgent surgery due to an adverse event, stroke, renal failure, wound infection, gastrointestinal complications, and atrial fibrillation (AF). Based on the greater efficacy of surgery, but a similar safety profile regarding "serious" adverse events demonstrated by the EVEREST II trial, it seems reasonable that the current updated guidelines recommend surgery over TEER in low-to-intermediate risk groups to treat severe primary MR. For surgical therapy, repair is definitely preferable over replacement whenever feasible given the risks associated with implanting prosthetic valves in cases such as infective endocarditis, thromboembolic complications, structural or non-structural valve dysfunction, and anticoagulation-related hemorrhage.

\section{TEER versus surgery in secondary mitral regurgitation}

For symptomatic secondary MR with a reduced left ventricular ejection fraction (LVEF), a separate decision-making flow is offered by the guidelines, in that further stratification is performed depending on the requirement for coronary revascularization. In cases where coronary revascularization is required, MV surgery is saved only for selected cases where coronary lesions are in need of coronary artery bypass grafting or for cases requiring other combined surgical procedures. Otherwise, percutaneous coronary intervention combined with TEER is suggested as the preferred option. This recommendation is perhaps grounded in the perceived high risks of surgery in cases of severe ischemic MR despite the lack of sufficient evidence in support of the overt clinical benefits of undertaking MV surgery in these patients [10-12]. Decision-making within surgical therapy is also complex with regards to choosing between repair and replacement, as well as selecting the repair technique - a downsized annuloplasty ring with or without adjunctive procedures on the sub-valvular apparatus or on the LV. The clinical implications of these procedural variables in surgical therapy have yet to be determined, and therefore, the guidelines remain conservative regarding surgical therapy in severe MR secondary to LV dysfunction.

Meanwhile TEER has emerged as a convincing modality in the management of secondary MR. Although the MITRA-FR trial (Multicentre Study of Percutaneous Mitral Valve Repair MitraClip Device in Patients with Severe Secondary Mitral Regurgitation) failed to demonstrate any significant benefits of TEER in reducing all-cause mortality and unplanned hospitalizations due to heart failure during 1 year post-intervention [13], the COAPT trial (Cardiovascular Outcomes Assessment of the MitraClip Percutaneous Therapy for Heart Failure Patients With Functional Mitral Regurgitation) showed significantly superior clinical outcomes in the 2-year rates of heart failure hospitalization (primary endpoint) and all-cause mortality (secondary endpoint) with TEER as compared with guideline-directed medical management [2]. On the basis of the COAPT trial findings, the Food and Drug Administration (FDA) approved the use of the MitraClip device (Abbott, Abbott Park, IL, USA) for the treatment of patients with symptomatic secondary MR ( $\geq$ moderately severe), reduced LV function (LVEF 20\%-50\%) and relatively preserved LV dimension (LV end-systolic diameter $<70 \mathrm{~mm}$ ) despite maximally tolerated guideline-directed medical therapies. With the same criteria, the Korean FDA approved the MitraClip device for use in the management of secondary MR in 2020.

On the basis of this background, TEER is the preferred option over surgery when an intervention is judged to be needed in cases of severe secondary MR. Meanwhile, surgery is saved only for cases where a transcatheter intervention is deemed inappropriate or for those in need of surgical coronary revascularization. However, this seemingly reasonable summary of the recommendations in fact needs careful interpretation. 


\section{Atrial versus ventricular functional mitral regurgitation}

The pathophysiologic mechanisms of secondary MR have clinical implications when it comes to the correction of MR by surgical or transcatheter interventions [14]. The 2 prototypes of functional MR-atrial and ventricular functional MR-are depicted in Fig. 1. Most patients with secondary MR have a dilated LV with wall motion abnormalities with consequent systolic tethering of the MV leaflets, while some of them exhibit regional wall motion abnormalities in the inferobasal or posterobasal segments even without global LV dysfunction and dilatation [15]. This entity fits the term "ventricular functional MR". Secondary MR may also occur by pure annular dilation in patients with severe left atrial (LA) dilation, which has been termed "atrial functional MR" [16]. Atrial functional MR is most commonly seen in patients with persistent or long-standing persistent $\mathrm{AF}$, and is associated with relative preservation of LV function and size. Despite its unique pathophysiology, atrial functional MR has received less attention; however, management recommendations need to take into account the mechanism of MR (AF) and the potential indications for rhythm control, as the guidelines denote $[8,14]$. In particular, catheter-based AF ablation would not be recommended in these patients, who are deemed to be appropriate candidates for surgical management of coexisting MR [17]. In this sense, surgical MV re-

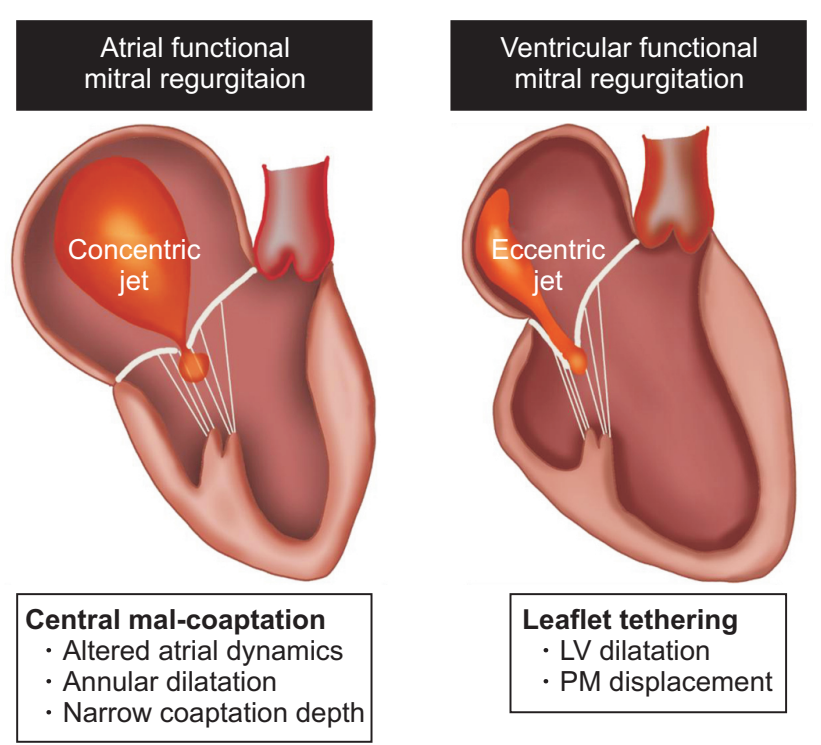

Fig. 1. Two types of secondary mitral regurgitation based on pathophysiologic mechanisms: atrial (A) and ventricular (B) functional mitral regurgitation. LV, left ventricle; PM, papillary muscle. pair combined with AF ablation seems to be the ideal option to treat atrial functional MR instead of transcatheter therapy (TEER with or without catheter-based AF ablation). There at least seems to be a need for separate decision-making trees within the entity of secondary MR, while the recent focused update mainly focused on cases occurring secondary to ventricular impairment, without sufficient detail on how to manage atrial functional MR as a distinct entity.

\section{Atrial functional mitral regurgitation: TEER or surgery?}

In a recent study that evaluated the outcomes of $74 \mathrm{pa}-$ tients undergoing TEER for functional MR, the authors demonstrated that baseline AF was associated with a significant reduction in survival rates over a period of 5 years, and multivariable analyses confirmed that AF was a significant and independent predictor of long-term mortality (hazard ration [HR], 2.65; 95\% confidence interval [CI], 1.12-6.47; $p=0.027$ ) [18]. This paper provides the insight that TEER may have limited value as a disease modifier in the presence of AF in cases where AF is a causal contributor to MR. In addition, from a technical standpoint, the primary driving force producing $\mathrm{MR}$ in atrial functional MR is annular dilatation, wherein 2 edges of the anterior and posterior leaflets are more displaced than in ventricular functional MR, and capturing these displaced edges with the device may be more challenging, posing a greater risk of treatment failure. More fundamentally, annular dilatation-the primary driver of atrial functional MR-is not addressed by TEER alone, and therefore additional annuloplasty (transcatheter) would be needed in some patients for more durable repair. The burden of procedures in this scenario, however, may exceed that of surgical therapy, as minimally-invasive surgical approaches have evolved in

Table 1. Factors favoring surgical therapy over transcatheter therapy in atrial functional mitral regurgitation

\section{Factors}

Relatively preserved left ventricular function to endure surgical risks Technical difficulty of transcatheter therapy in capturing displaced leaflet edges

Effective mitral ring annuloplasty available

Effective surgical ablation against atrial fibrillation available

Concomitant tricuspid repair for advanced tricuspid regurgitation available

Minimally invasive approaches available for all of the above procedures at once 
their own right to offer more comfortable and earlier postoperative recovery.

\section{Lessons from surgical studies: insights into ablation of atrial fibrillation}

Chen and colleagues retrospectively analyzed the surgical outcomes of 82 patients with atrial functional MR, all of whom had AF at baseline [19]. Among these patients undergoing surgical MV repair, $63.4 \%$ underwent concomitant surgical AF ablation. Of note, freedom from recurrent MR after surgery was significantly superior in those undergoing AF ablation compared with patients who did not receive $\mathrm{AF}$ ablation, with 3-year rates of $93.8 \%$ versus $44.2 \%$ $(\mathrm{p}=0.035)$. In addition, the outcome gap in MR recurrence between these 2 groups was even more prominent in patients with smaller LAs $(\leq 60 \mathrm{~mm})$. These findings indicate that ablation of AF may also contribute to more durable MV function in the long-term following MV repair in atrial functional MR.

Compelling evidence has also been reported from larger observational studies that concomitant ablation of AF during MV surgery is associated with improved survival and decreased risks of stroke and heart failure [20-24]. For instance, in a study involving 1,229 patients undergoing rheumatic MV surgery in the presence of AF, concomitant AF ablation resulted in far superior rhythm outcomes (freedom from AF at 5 years, $76.5 \%$ versus $5.0 \% ; \mathrm{p}<0.001$ ), reduced overall mortality (HR, 0.62; 95\% CI, 0.46-0.83; $\mathrm{p}=0.002)$ and a lower incidence of systemic thromboembolic events (HR, 0.49; 95\% CI, 0.32-0.76; $\mathrm{p}=0.001$ ) during long-term follow-up, as demonstrated by rigorous adjustments for pre-specified variables by propensity score modeling [25]. In another study, surgical AF ablation had the strongest impact on preventing tricuspid regurgitation (TR) progression over any other factors among 1,208 patients with mild baseline TR undergoing MV surgery [26]. This preventive effect of AF ablation against the progression of TR has also been demonstrated in a number of surgical studies $[20,21,27]$. These study results augment the body of evidence showing that concomitant AF ablation is important for restore and maintaining tricuspid valve function. This is an important notion in atrial functional MR because advanced TR is found in the vast majority of these patients, and the decision-making on how to manage TR should also be considered in relation to concomitant AF ablation.

Taking the knowledge cultivated from surgical studies into consideration, surgical therapy is probably the prefera- ble treatment option in atrial functional MR, saving TEER as only indicated in high-risk or inoperable patients. In other words, the term "severe secondary MR" should not automatically be perceived as an indication for TEER. Notably, the early surgical outcomes of atrial functional MR seem acceptable, given relatively preserved LV function, relative to those in patients with MR caused by LV dysfunction. As LV systolic dysfunction is a well-known risk factor for postoperative adverse outcomes, the surgical risk may be tolerable in the majority of patients presenting with atrial functional MR [28]. Table 1 summarizes the potential strengths of surgical therapy over TEER for severe atrial functional MR. Most importantly, the decision to undertake surgery or TEER should be based on an individualized approach, and shared decision-making with discussions within the heart team is the ideal pathway in the management of MR.

\section{Conclusion}

In conclusion, surgery seems to be preferable over TEER in primary MR that needs intervention, while the decision-making for secondary MR seems to need further stratification depending on the pathophysiologic mechanism of MR, as TEER is generally the more suitable option in ventricular functional MR, while atrial functional MR may be best treated by surgical MV repair combined with $\mathrm{AF}$ ablation in the majority of patients. This particular issue, however, needs further research to offer scientific evidence to guide optimal management strategies.

\section{Conflict of interest}

No potential conflict of interest relevant to this article was reported.

\section{ORCID}

Joon Bum Kim: https://orcid.org/0000-0001-5801-2395

\section{References}

1. Feldman T, Foster E, Glower DD, et al. Percutaneous repair or surgery for mitral regurgitation. N Engl J Med 2011;364:1395-406.

2. Stone GW, Lindenfeld J, Abraham WT, et al. Transcatheter mitral-valve repair in patients with heart failure. N Engl J Med 2018; 379:2307-18

3. Leon MB, Smith CR, Mack M, et al. Transcatheter aortic-valve implantation for aortic stenosis in patients who cannot undergo surgery. 
N Engl J Med 2010;363:1597-607.

4. Mack MJ, Leon MB, Thourani VH, et al. Transcatheter aortic-valve replacement with a balloon-expandable valve in low-risk patients. $\mathrm{N}$ Engl J Med 2019;380:1695-705.

5. Popma JJ, Deeb GM, Yakubov SJ, et al. Transcatheter aortic-valve replacement with a self-expanding valve in low-risk patients. N Engl J Med 2019;380:1706-15.

6. Smith CR, Leon MB, Mack MJ, et al. Transcatheter versus surgical aortic-valve replacement in high-risk patients. N Engl J Med 2011; 364:2187-98.

7. Elbadawi A, Elgendy IY, Mohamed AH, et al. Temporal trends and outcomes of transcatheter mitral valve repair and surgical mitral valve intervention. Cardiovasc Revasc Med 2020;21:1560-6.

8. Bonow RO, O'Gara PT, Adams DH, et al. 2020 Focused update of the 2017 ACC expert consensus decision pathway on the management of mitral regurgitation: a report of the American College of Cardiology Solution Set Oversight Committee. J Am Coll Cardiol 2020;75:2236-70.

9. Writing Committee Members, Otto CM, Nishimura RA, et al. 2020 ACC/AHA guideline for the management of patients with valvular heart disease: executive summary: a report of the American College of Cardiology/American Heart Association Joint Committee on clinical practice guidelines. J Am Coll Cardiol 2021;77:450-500.

10. Wu AH, Aaronson KD, Bolling SF, Pagani FD, Welch K, Koelling TM. Impact of mitral valve annuloplasty on mortality risk in patients with mitral regurgitation and left ventricular systolic dysfunction. J Am Coll Cardiol 2005;45:381-7.

11. Goldstein D, Moskowitz AJ, Gelijns AC, et al. Two-year outcomes of surgical treatment of severe ischemic mitral regurgitation. $\mathrm{N}$ Engl J Med 2016;374:344-53.

12. Michler RE, Smith PK, Parides MK, et al. Two-year outcomes of surgical treatment of moderate ischemic mitral regurgitation. N Engl J Med 2016;374:1932-41.

13. Obadia JF, Messika-Zeitoun D, Leurent G, et al. Percutaneous repair or medical treatment for secondary mitral regurgitation. $\mathrm{N}$ Engl J Med 2018;379:2297-306.

14. Deferm S, Bertrand PB, Verbrugge FH, et al. Atrial functional mitral regurgitation: JACC review topic of the week. J Am Coll Cardiol 2019;73:2465-76.

15. Kwan J, Shiota T, Agler DA, et al. Geometric differences of the mitral apparatus between ischemic and dilated cardiomyopathy with significant mitral regurgitation: real-time three-dimensional echocardiography study. Circulation 2003;107:1135-40.
16. Gertz ZM, Raina A, Saghy L, et al. Evidence of atrial functional mitral regurgitation due to atrial fibrillation: reversal with arrhythmia control. J Am Coll Cardiol 2011;58:1474-81.

17. Calkins H, Hindricks G, Cappato R, et al. 2017 HRS/EHRA/ECAS/ APHRS/SOLAECE expert consensus statement on catheter and surgical ablation of atrial fibrillation: executive summary. Heart Rhythm 2017;14:e445-94.

18. Baldi C, Citro R, Silverio A, et al. Predictors of outcome in heart failure patients with severe functional mitral regurgitation undergoing MitraClip treatment. Int J Cardiol 2019;284:50-8.

19. Chen J, Wang Y, Lv M, et al. Mitral valve repair and surgical ablation for atrial functional mitral regurgitation. Ann Transl Med 2020; $8: 1420$.

20. Kim JB, Moon JS, Yun SC, et al. Long-term outcomes of mechanical valve replacement in patients with atrial fibrillation: impact of the maze procedure. Circulation 2012;125:2071-80.

21. Yoo JS, Kim JB, Jung SH, Choo SJ, Chung CH, Lee JW. Impact of the maze procedure and postoperative atrial fibrillation on progression of functional tricuspid regurgitation in patients undergoing degenerative mitral repair. Eur J Cardiothorac Surg 2013;43:520-5.

22. Kim HJ, Kim JB, Jung SH, et al. Surgical ablation of atrial fibrillation in patients with a giant left atrium undergoing mitral valve surgery. Heart 2016;102:1206-14.

23. Kim WK, Kim HJ, Kim JB, et al. Clinical outcomes in 1731 patients undergoing mitral valve surgery for rheumatic valve disease. Heart 2018;104:841-8.

24. Pyo WK, Kim HJ, Kim JB, et al. Does surgical ablation of atrial fibrillation benefit patients undergoing bioprosthetic valve replacement? Semin Thorac Cardiovasc Surg 2021 Jun 6 [Epub]. https://doi. org/10.1053/j.semtcvs.2021.05.015.

25. Kim WK, Kim HJ, Kim JB, et al. Concomitant ablation of atrial fibrillation in rheumatic mitral valve surgery. J Thorac Cardiovasc Surg 2019;157:1519-28.

26. Kim WK, Kim SE, Yoo JS, et al. Impact of valve repair on mild tricuspid insufficiency in rheumatic mitral surgery. J Thorac Cardiovasc Surg 2021 Jun 2 [Epub]. https://doi.org/10.1016/j.jtcvs.2021.05.033.

27. Ro SK, Kim JB, Jung SH, Choo SJ, Chung CH, Lee JW. Mild-tomoderate functional tricuspid regurgitation in patients undergoing mitral valve surgery. J Thorac Cardiovasc Surg 2013;146:1092-7.

28. Kim HJ, Kim JB, Kim SO, et al. A risk prediction model for operative mortality after heart valve surgery in a Korean cohort. J Chest Surg 2021;54:88-98. 